\title{
Geometry of Bend: Singular Lines and Defects in Twist-Bend Nematics
}

\author{
Jack Binysh $\odot,{ }^{1,2, *}$ Joseph Pollard, ${ }^{1, *}$ and Gareth P. Alexander $\oplus^{3, \dagger}$ \\ ${ }^{1}$ Mathematics Institute, Zeeman Building, University of Warwick, Coventry CV4 7AL, United Kingdom \\ ${ }^{2}$ Department of Physics, University of Bath, Claverton Down, Bath BA2 7AY, United Kingdom \\ ${ }^{3}$ Department of Physics and Centre for Complexity Science, University of Warwick, Coventry CV4 7AL, United Kingdom
}

(Received 3 February 2020; revised 25 May 2020; accepted 15 June 2020; published 24 July 2020)

\begin{abstract}
We describe the geometry of bend distortions in liquid crystals and their fundamental degeneracies, which we call $\beta$ lines; these represent a new class of linelike topological defect in twist-bend nematics. We present constructions for smecticlike textures containing screw and edge dislocations and also for vortexlike structures of double twist and Skyrmions. We analyze their local geometry and global structure, showing that their intersection with any surface is twice the Skyrmion number. Finally, we demonstrate how arbitrary knots and links can be created and describe them in terms of merons, giving a geometric perspective on the fractionalization of Skyrmions.
\end{abstract}

DOI: 10.1103/PhysRevLett.125.047801

Fresh perspectives invariably accompany the discovery of a new phase: The experimental discovery of the twistbend nematic phase [1-3] invites fresh consideration of nematic geometry and topology. The twist-bend nematic is a fluid mesophase in which the nematic orientation exhibits a heliconical modulation with nanoscale pitch and modest cone angle [4]. It occurs in bent core compounds (banana molecules) and is characterized by a state of nonzero bend $[5,6]$. The geometrical and topological features of bend are thus a natural vehicle for describing the degeneracies and defects of the twist-bend phase, however, they arise more generally and apply to any material described by a unit vector, or line, field.

Geometric elastic distortions pervade soft matter physics [7], providing a common conceptual framework as well as numerous methods-including boundary conditions, substrate topography, and surface curvature-for designing properties or functionality [8-16]. Geometric methods also relate to topological properties through the Gauss-Bonnet theorem and Berry phase physics, giving them greater potential for material control. A common feature of many materials are degeneracies along lines or curves, with examples including flux lines in superconductors [17], fluid vortices [18], nodal lines in optical beams [19], C lines in electromagnetic fields [20], defect lines in liquid crystals [21,22], and umbilic lines in general [23]. In many instances these lines are fundamental to the organization and properties of the entire material, simultaneously

Published by the American Physical Society under the terms of the Creative Commons Attribution 4.0 International license. Further distribution of this work must maintain attribution to the author(s) and the published article's title, journal citation, and DOI. characterizing it and offering a mechanism for controlling and engineering specific responses.

In this Letter, we introduce a linelike geometric degeneracy associated to zeros of the bend in a unit vector field, that we call $\beta$ lines. These occur in all materials with vector or orientational order, such as liquid crystals and ferromagnets, but have added significance when there is an energetic preference for nonzero bend, and in such materials $\beta$ lines are a topological defect. We give constructions of both smecticlike textures and Skyrmion states in twist-bend nematics and characterize them in terms of their $\beta$ lines. We describe the local structure of generic $\beta$ lines and show that their intersection number with a surface gives a Skyrmion count. Finally, we show how complex textures encoding knotted $\beta$ lines may be constructed, analogous to the "heliknotons" recently created in cholesterics [24], and characterize them in terms of merons. Additional illustrations and examples, as well as further technical details, are presented in the Supplemental Material [25].

Orientational order is described by a unit vector $\mathbf{n}$, called the director. Nematic symmetry, $\mathbf{n} \sim-\mathbf{n}$, corresponds to linelike alignment, rather than vectorial. The bend $\mathbf{b}=$ $(\mathbf{n} \cdot \nabla) \mathbf{n}=-\mathbf{n} \times(\nabla \times \mathbf{n})$ is the curvature of the director integral curves; it is a globally defined vector whose sign does not reverse under $\mathbf{n} \rightarrow-\mathbf{n}$. As $\mathbf{n}$ is a unit vector the bend is everywhere orthogonal to it, $\mathbf{b} \cdot \mathbf{n}=0$. Thus, although bend is a vector field in three-dimensional space, it is atypical, having only 2 degrees of freedom. In particular, the set of points where it vanishes-the inflectional points of the integral curves-is one dimensional, and forms a collection of fundamental curves in the material that are characteristic of it; we call them $\beta$ lines.

A model system for exploring $\beta$ lines is the twist-bend nematic. It may be described by a Frank free energy with negative bend elastic constant [5], or by coupling the bend 


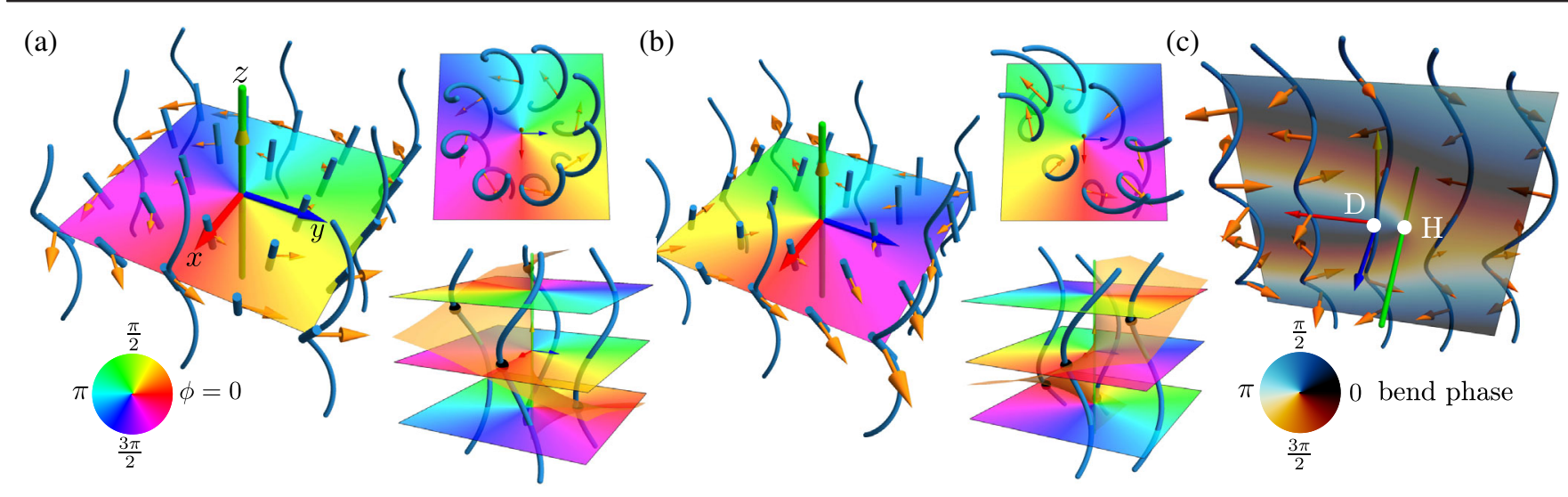

FIG. 1. Examples of $\beta$ lines in smecticlike twist-bend singularities. Director and its integral curves shown in blue, with the bend vector in orange and $\beta$ lines in green. (a), (b) Screw dislocations in the helical phase of the twist-bend ground state, with strength $s=+1$ (a) and $s=-1$ (b). Color indicates the phase $\phi$ from Eq. (2). Insets show the winding of the helical phase around the $\beta$ line from multiple perspectives. Orange surfaces in the bottom inset show $\phi=0$ with points of equal phase along the integral curves marked by black dots. (c) Edge dislocation in $\phi$; color indicates the $x y$ angle of the bend vector. In passing from negative to positive $x$ the director integral curves pick up an extra turn, implying the existence of a $\beta$ line. The $\beta$ line location is not the phase singularity in $\phi(D)$, but the hyperbolic point $H$ where $\nabla \phi$ vanishes.

to a vector polarization $\mathbf{p}$, coming from the "banana" shape of the molecules, with a free energy [6],

$F=\int \frac{K}{2}|\nabla \mathbf{n}|^{2}-\lambda \mathbf{b} \cdot \mathbf{p}+\frac{C}{2}|\nabla \mathbf{p}|^{2}+\frac{U}{4}\left(1-|\mathbf{p}|^{2}\right)^{2} d V$,

where $K$ is a Frank elastic constant, $\lambda$ is a coupling constant, $C$ is an elastic constant for the polarization, and $U$ sets the scale of the bulk ordering energy. This favors the heliconical director $\mathbf{n}=\cos \theta \mathbf{e}_{z}+\sin \theta\left(\cos q z \mathbf{e}_{x}+\sin q z \mathbf{e}_{y}\right)$, with the conical angle $\theta$ and helical wave vector $q$ determined by the elastic moduli $[4,6]$. The integral curves are helices of constant curvature and torsion; the bend $\mathbf{b}=$ $q \sin \theta \cos \theta\left(-\sin q z \mathbf{e}_{x}+\cos q z \mathbf{e}_{y}\right)$ has constant magnitude and rotates at the same rate as the director. We review the geometry of the heliconical director and the free energy (1) in the Supplemental Material [25], which includes Refs. [26$31]$. On scales large compared to the helical pitch $(2 \pi / q)$ the twist-bend phase has the same elastic energy as a smectic [32-35] and exhibits all the same features, textures, and defects, despite there being no mass-density wave. These smecticlike defects are all associated with $\beta$ lines.

We consider first screw dislocations in the helical integral curves of the twist-bend ground state, Fig. 1. Here, the phase of the helices winds by $2 \pi s$ on a circle enclosing the screw axis, where $s$ is the integer dislocation strength; we show $s=+1$ in Fig. 1(a) and $s=-1$ in Fig. 1 (b). There is the same winding number in the bend (orange arrows), guaranteeing the existence of a $\beta$ line. These textures are captured by the director field

$$
\mathbf{n}=\cos \theta \mathbf{e}_{z}+\sin \theta\left[\cos \phi \mathbf{e}_{x}+\sin \phi \mathbf{e}_{y}\right],
$$

where $\phi=q z+s \arctan (y / x)$ and the cone angle $\theta$ varies smoothly from its far field preferred value to vanish on the $z$ axis. The integral curves degenerate to a straight line along the $z$ axis which, having no curvature, is a $\beta$ line. Using instead $\phi=q z+s \arctan (z / x)$ yields an edge dislocation, Fig. 1(c). Here the $\beta$ line does not coincide with the dislocation itself, where $\phi$ is singular $[y$ axis, marked $D$ in Fig. 1(c)], but instead it is displaced to one side, at the position of the hyperbolic point [ $H$ in Fig. 1(c)], where $\nabla \phi$ is zero [36]. A detailed comparison of the winding of $\phi$ and its singularities versus that of the bend vector is given in the Supplemental Material [25], which contains Ref. [37]. These examples can be set in a general context: For a smectic phase field $\phi$ with layer normal $\mathbf{N}$ we set $\mathbf{n}=\cos \theta \mathbf{N}+\sin \theta\left[\cos \phi \mathbf{e}_{1}+\sin \phi \mathbf{e}_{2}\right]$, where $\mathbf{e}_{1}, \mathbf{e}_{2}$ are an orthonormal basis for the planes orthogonal to $\mathbf{N}$ that is parallel transported along it, $\left(\nabla_{\mathbf{N}} \mathbf{e}_{1}\right) \cdot \mathbf{e}_{2}=0$. The singularities in the smectic phase field then all induce $\beta$ lines in the director. In the Supplemental Material [25], we provide examples for twist-grain-boundaries [38-40] and parabolic focal conics [41,42].

A separate set of examples of $\beta$ lines is provided by the vortex structures familiar from cholesterics and Skyrmions. A canonical example is the double twist profile $\mathbf{n}=$ $\cos q \rho \mathbf{e}_{z}+\sin q \rho \mathbf{e}_{\phi}$ shown in Fig. 2(a), for which the bend is radial, $\mathbf{b}=-(1 / \rho) \sin ^{2} q \rho \mathbf{e}_{\rho}$, and vanishes linearly along the axis with winding number +1 . Although the winding number is the same as the $s=+1$ screw dislocation, Fig. 1(a), the structure is distinct; each helical integral curve encircles the $\beta$ line, in contrast to the screw dislocation where they do not. These two $\beta$ lines are therefore topologically distinct, in the sense that one cannot convert one into the other without creating additional $\beta$ lines. Such a double twist cylinder occurs at the core of a Skyrmion embedded in a twist-bend background, Figs. 2(b), 2(c); Skyrmions are nonsingular field configurations in cholesterics and 


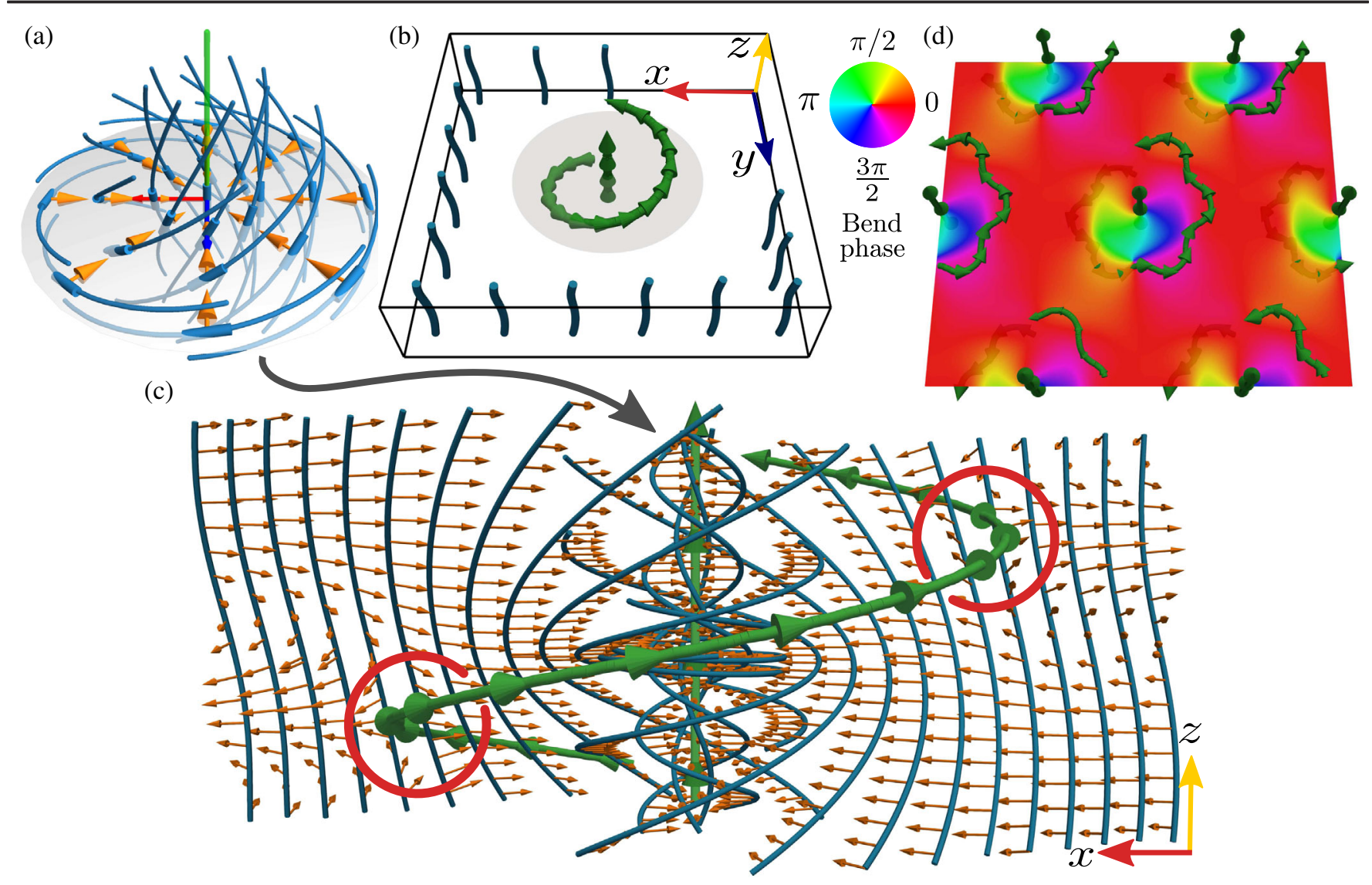

FIG. 2. (a) $\beta$ line at the center of a double twist cylinder-the integral curves wind about the $\beta$ line, making this +1 defect topologically distinct from the screw dislocation of Fig. 1(a). (b) A single Skyrmion (gray disc) embedded in a heliconical background. There are two co-oriented $\beta$ lines with orientations indicated by a choice of tangent vector, and agreeing with the far field heliconical director having negative $z$ component. (c) Detailed structure of the director integral curves (blue), bend vector (orange), and $\beta$ lines (green) of a twist-bend Skyrmion. Red circles highlight the winding of the bend vector about the second, helical, $\beta$ line. (d) A hexagonal lattice of twist-bend Skyrmions, with midplane colored by the $x y$ angle of the bend vector. Simulation results in panels (b),(c),(d) are shown for $\theta=0.1, U / C=0.3$.

ferromagnets with Dzyaloshinskii-Moriya interaction [4347], which carry a topological charge $Q=(1 / 4 \pi) \int \mathbf{n}$. $\partial_{x} \mathbf{n} \times \partial_{y} \mathbf{n} d x d y$. The analogous helical director structures suggest it is possible Skyrmions also arise in twist-bend nematics, and indeed we find them to be (meta)stable in simulation, Fig. 2(d). The Skyrmion contains two $\beta$ lines [Figs. 2(b), 2(c)], one along the central axis with the structure of the double twist cylinder, Fig. 2(a), and the second a helix with pitch equal to that of the heliconical far field director and winding number of the bend -1 . These $\beta$ lines are a topological necessity and count the Skyrmion charge $Q$; we demonstrate below (and provide further detail in the Supplemental Material [25]) that there are two $\beta$ lines per Skyrmion. A full Skyrmion phase diagram, analogous to that constructed for cholesterics [48], would be of clear interest, although it is not the focus of this Letter; here, we simply note that we have confirmed (meta)stability for the heliconical far field angle $\theta \in[0.1,0.5], U / C \in[0.1,0.5]$, in simulations performed using periodic boundary conditions with box height chosen to match one pitch length ( $2 \pi$ rotation) of the twist-bend director. The stability we have seen suggests that twist-bend Skyrmions could be directly nucleated by adapting techniques used in cholesteric cells or in magnetic systems.

Thus far we have discussed $\beta$ lines in particular examples motivated by twist-bend nematics and cholesterics. We now turn to a general description of their geometry and topological significance. In our simple examples, the director $\mathbf{n}$ is either collinear with the $\beta$ line tangent $\mathbf{t}$, as in Figs. 1(a), 1(b), and 2(a), or orthogonal to it as in Fig. 1(c). Generically neither is the case, and $\mathbf{n}$ and $\mathbf{t}$ make some intermediate angle. Points where they are orthogonal have codimension 1 and are called Legendrian (see, for example, Ref. [49]); points of collinearity are codimension 2 and do not occur except in situations of high symmetry. A local description of a generic point on a $\beta$ line can be developed by introducing adapted coordinates $\mathbf{n} \approx n_{x} \mathbf{e}_{x}+$ $n_{y} \mathbf{e}_{y}+\mathbf{e}_{z}$ and a Taylor series 

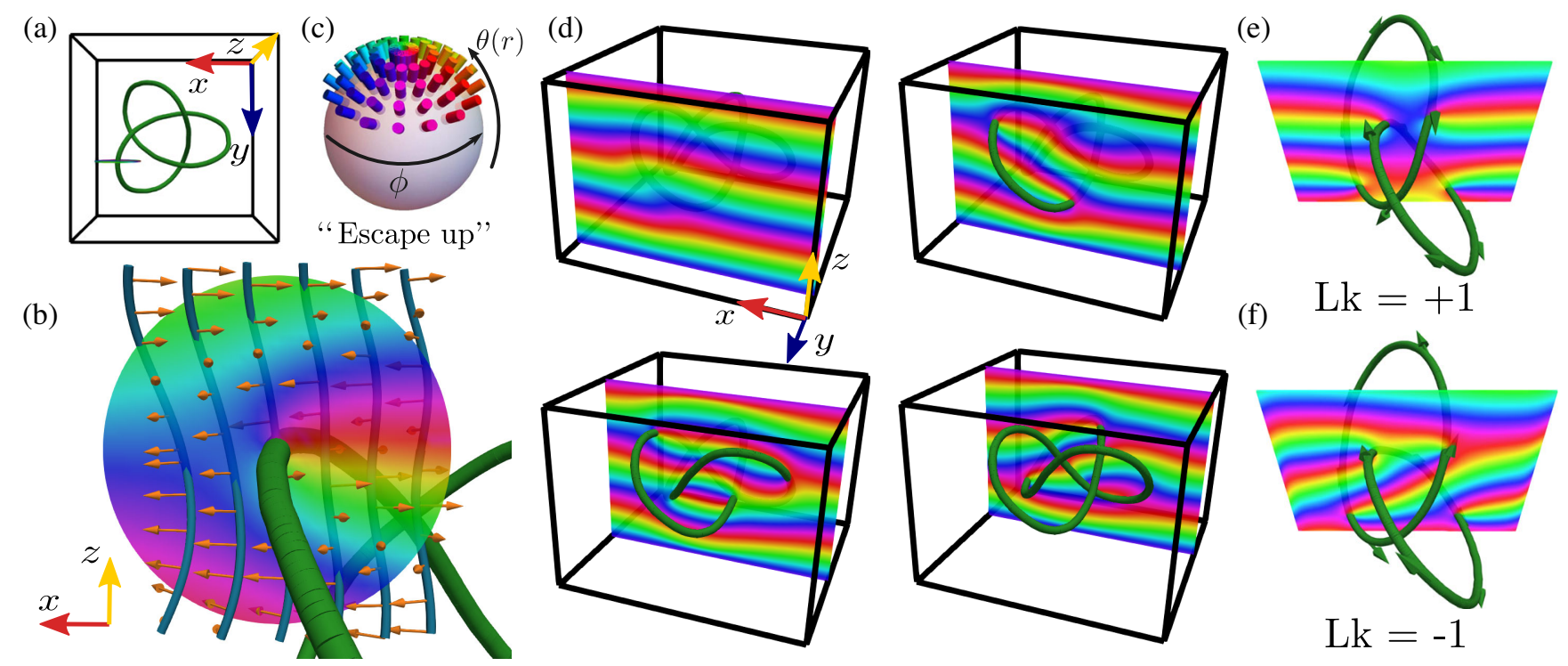

FIG. 3. Knotted $\beta$ line meron textures in twist-bend nematics. (a) $\beta$ line tied into a trefoil knot, embedded in the heliconical background, with a cross-sectional slice marked and shown in (b). The local structure of the director (b), (c) is an escape-up meron containing a single $\beta$ line (color denotes bend phase; same as in Fig. 2). The director in the cross section shown in (b) covers the top of the unit sphere as shown in (c), corresponding to escape up. In (d) we show the $\beta$ line from the side, with the bend phase (color) on several cross-sectional slices. (e), (f) Hopf links with linking numbers +1 (e) and -1 (f), and their distinct phase fields.

$$
\begin{gathered}
{\left[\begin{array}{l}
n_{x} \\
n_{y}
\end{array}\right]=\left[\left.\nabla_{\perp} \mathbf{n}\right|_{0}+\left.z\left(\partial_{z} \nabla_{\perp} \mathbf{n}\right)\right|_{0}\right]\left[\begin{array}{l}
x \\
y
\end{array}\right]+\frac{1}{2} z^{2}\left[\begin{array}{l}
s_{x} \\
s_{y}
\end{array}\right],} \\
{\left[\begin{array}{l}
b_{x} \\
b_{y}
\end{array}\right]=\left[\left(\left.\nabla_{\perp} \mathbf{n}\right|_{0}\right)^{2}+\left.\partial_{z} \nabla_{\perp} \mathbf{n}\right|_{0}\right]\left[\begin{array}{l}
x \\
y
\end{array}\right]+z\left[\begin{array}{l}
s_{x} \\
s_{y}
\end{array}\right],}
\end{gathered}
$$

retaining only terms that contribute at linear order to the bend.

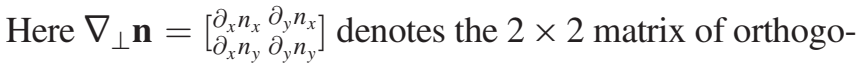
nal gradients of the director [23] and $\partial_{z} \nabla_{\perp} \mathbf{n}$ is its rate of change along the local director; $\left[s_{x}, s_{y}\right]$ controls the angle between $\mathbf{t}$ and $\mathbf{n}$. The winding number in the $x y$ plane is \pm 1 according to the sign of $\operatorname{det}\left[\left(\left.\nabla_{\perp} \mathbf{n}\right|_{0}\right)^{2}+\left.\partial_{z} \nabla_{\perp} \mathbf{n}\right|_{0}\right]$. When the derivatives $\left.\partial_{z} \nabla_{\perp} \mathbf{n}\right|_{0}$ are negligible this reduces to $\left(\left.\operatorname{det} \nabla_{\perp} \mathbf{n}\right|_{0}\right)^{2}$ and the winding is always +1 , so that the different profiles of $\beta$ lines are controlled crucially by the parallel derivatives of the orthogonal director gradients. In the Supplemental Material [25], which includes Refs. [50,51], we use Eq. (3), (4) to construct a variety of $\beta$ lines with different local profiles, including a Legendrian point.

We now describe the global structure beginning with a canonical orientation of $\beta$ lines via the operator $\nabla \mathbf{b}$. Along the $\beta$ lines there are two canonical frames; the tangent vector to the curve $\mathbf{t}$ and normal plane $\nu$, and the director $\mathbf{n}$ and its normal plane $\xi$. $\nabla \mathbf{b}$ defines an isomorphism $\nu \rightarrow \xi$, detailed in the Supplemental Material [25], and we orient the $\beta$ line such that this isomorphism preserves orientation. We note that the orientation obtained reverses upon $\mathbf{n} \rightarrow-\mathbf{n}$, which corresponds to the change in sign of point defects (or Skyrmion charge) under the same replacement [52]. On the complement of the $\beta$ lines there is the FrenetSerret framing of the integral curves. The $\beta$ lines are singularities of this framing. We write $\mathbf{b}=\kappa \mathbf{e}_{1}$, with $\kappa$ the curvature of the integral curves, and $\mathbf{e}_{2}=\mathbf{n} \times \mathbf{e}_{1}$. This framing yields a connection 1-form $\omega=\left(\nabla \mathbf{e}_{1}\right) \cdot \mathbf{e}_{2}$ for the plane field $\xi$. The component of $\omega$ along the director is the torsion $\tau=\omega(\mathbf{n})=\left(\nabla_{\mathbf{n}} \mathbf{e}_{1}\right) \cdot \mathbf{e}_{2}$, while the vector dual to it is the local pitch axis of the heliconical twist-bend state. For example, the smectic-based twist-bend director (2) has connection 1-form $\omega=\cos \theta d \phi$; the torsion is $\tau \approx q \cos ^{2} \theta$ and the pitch axis is along $\nabla \phi$. Topological information is conveyed by the associated curvature 2 -form $\Omega=$ $-\sin \theta d \theta \wedge d \phi=(-1 / 2) \epsilon_{i j k} n_{i} d n_{j} \wedge d n_{k}$. Given a surface $S$, the $\beta$ lines intersect it in a set of points $p_{i}$ and by the Gauss-Bonnet-Chern theorem,

$$
\frac{1}{2 \pi} \int_{\partial S} \omega-\frac{1}{2 \pi} \int_{S} \Omega=\sum_{i} \operatorname{Int}_{p_{i}}(\beta, S),
$$

where $\operatorname{Int}_{p_{i}}(\beta, S)$ denotes the signed intersection number at point $p_{i}$ of an oriented $\beta$ line with an oriented surface $S$. For Skyrmion textures this total intersection number is $2 Q$, giving two $\beta$ lines per Skyrmion as seen in Fig. 2. In the Supplemental Material [25], which contains also Refs. [5357], we give detailed applications of Eq. (5) to the examples of the screw dislocation (Fig. 1), the double twist cylinder [Fig. 2(a)], and the Skyrmion texture [Figs. 2(b), 2(c)], in each case demonstrating the topological necessity of the $\beta$ lines present. 
Finally, we discuss fully three-dimensional textures and show that it is possible to embed an arbitrary knotted or linked set of $\beta$ lines into a heliconical background, via an extension of our constructions for screw and edge dislocations. Given any knot or link $K$, the director

$$
\mathbf{n}=\cos \theta \mathbf{e}_{z}+\sin \theta\left[\cos \phi_{K} \mathbf{e}_{x}+\sin \phi_{K} \mathbf{e}_{y}\right],
$$

where $\phi_{K}=q z+\frac{1}{2} \omega_{K}$, with $\omega_{K}$ the solid angle function for $K$ [58], embeds a helical winding of the director integral curves around a tubular neighborhood of $K$, Fig. 3; as before, the cone angle $\theta$ should be made to vary from its far field value to vanish along $K$. The phase winding in the helical integral curves guarantees the existence of a $\beta$ line. Examples for the trefoil knot, Figs. 3(a)-3(d), and Hopf link, Figs. 3(e), 3(f), are shown.

The director texture around the knot is that of a meron tube extruded along $K$. A meron is a fractionalization of a Skyrmion that carries half the topological charge [46,59]. $\beta$ lines provide a natural geometric perspective on this fractionalization: since each Skyrmion comprises two $\beta$ lines, a single $\beta$ line represents half a Skyrmion, i.e., a meron. In terms of the heliconical phase field $\phi_{K}$, these meron tubes are edge dislocations where heliconical layers terminate. Exactly these structures were recently created experimentally in cholesteric cells and shown to form highly controllable and responsive knotted solitons [24]. In that experiment, links of "escape up" and "escape down" meron tubes combined to give nonzero Hopf invariant. For the twist-bend nematic phase, the small conical angle $\left(\theta \approx 25^{\circ}[3]\right)$ gives an energetic preference to escape up merons over escape down, whereas in cholesterics $(\theta=\pi / 2)$ the two types of meron are degenerate. Even with only escape up merons, where the Hopf invariant is trivial, the linking is still a relevant quantity with distinct textures for different values of the linking number, $\operatorname{Lk}\left(\beta_{i}, \beta_{j}\right)$. In Figs. 3(e), 3(f) we show examples for the Hopf link with linking numbers \pm 1 where the layer structure through the middle of the link is different in the two cases; further images of an unknot, the trefoil, and both types of Hopf link are given in the Supplemental Material [25], which contains Refs. [60,61].

We have given an initial description of geometric degeneracies in the bend of a vector field, which we call $\beta$ lines, and their connection to topological features, including smectic singularities, Skyrmions, and merons. We have couched the majority of the discussion around the twist-bend nematic phase, in which the $\beta$ lines are novel topological defects, however the same structures arise in any orientationally ordered material. As one example in a different setting, active materials with extensile activity exhibit a bend driven instability in (three-dimensional) active nematics and cholesterics [62-64] and so naturally exist in states with nonzero bend distortion. The geometric degeneracies we have introduced here will also arise there and may provide a means for their analysis.

This work was supported by the UK EPSRC through Grant No. EP/L015374/1. J. B. supported by a Warwick IAS Early Career Fellowship.

*These authors contributed equally to this work.

†.P.Alexander@warwick.ac.uk

[1] M. Cestari et al., Phase behavior and properties of the liquid-crystal dimer 1", 7"'-bis(4-cyanobiphenyl-4'-yl) heptane: A twist-bend nematic liquid crystal, Phys. Rev. E 84, 031704 (2011).

[2] V. Borshch et al., Nematic twist-bend phase with nanoscale modulation of molecular orientation, Nat. Commun. 4, 2635 (2013).

[3] D. Chen et al., Chiral heliconical ground state of nanoscale pitch in a nematic liquid crystal of achiral molecular dimers, Proc. Natl. Acad. Sci. U.S.A. 110, 15931 (2013).

[4] A. Jákli, O. D. Lavrentovich, and J. V. Selinger, Physics of liquid crystals of bent-shaped molecules, Rev. Mod. Phys. 90, 045004 (2018).

[5] I. Dozov, On the spontaneous symmetry breaking in the mesophases of achiral banana-shaped molecules, Europhys. Lett. 56, 247 (2001).

[6] S. Shamid, S. Dhakal, and J. V. Selinger, Statistical mechanics of bend flexoelectricity and the twist-bend phase in bent-core liquid crystals, Phys. Rev. E 87, 052503 (2013).

[7] R. D. Kamien, The geometry of soft materials: A primer, Rev. Mod. Phys. 74, 953 (2002).

[8] V. Vitelli and W. T. M. Irvine, The geometry and topology of soft materials, Soft Matter 9, 8086 (2013).

[9] L. Tran, M. O. Lavrentovich, D. A. Beller, N. Li, K. J. Stebe, and R. D. Kamien, Lassoing saddle splay and the geometrical control of topological defects, Proc. Natl. Acad. Sci. U.S.A. 113, 7106 (2016).

[10] G. Napoli and L. Vergori, Extrinsic Curvature Effects on Nematic Shells, Phys. Rev. Lett. 108, 207803 (2012).

[11] E. A. Matsumoto, D. A. Vega, A. D. Pezzutti, N. A. García, P. M. Chaikin, and R. A. Register, Wrinkling and splay conspire to give positive disclinations negative curvature, Proc. Natl. Acad. Sci. U.S.A. 112, 12639 (2015).

[12] G. T. Vu, A. A. Abate, L. R. Gómez, A. D. Pezzutti, R. A. Register, D. A. Vega, and F. Schmid, Curvature as a Guiding Field for Patterns in Thin Block Copolymer Films, Phys. Rev. Lett. 121, 087801 (2018).

[13] P. W. Ellis, K. Nayani, J. P. McInerney, D. Z. Rocklin, J. O. Park, M. Srinivasarao, E. A. Matsumoto, and A. FernandezNieves, Curvature-Induced Twist in Homeotropic Nematic Tori, Phys. Rev. Lett. 121, 247803 (2018).

[14] T. J. White and D. J. Broer, Programmable and adaptive mechanics with liquid crystal polymer networks and elastomers, Nat. Mater. 14, 1087 (2015).

[15] C. Mostajeran, M. Warner, and C. D. Modes, Frame, metric and geodesic evolution in shape-changing nematic shells, Soft Matter 13, 8858 (2017).

[16] H. Aharoni, Y. Xia, X. Zhang, R. D. Kamien, and S. Yang, Universal inverse design of surfaces with thin nematic 
elastomer sheets, Proc. Natl. Acad. Sci. U.S.A. 115, 7206 (2018).

[17] A. A. Abrikosov, On the magnetic properties of superconductors of the second group, Zh. Eksp. Teor. Fiz. 32, 1442 (1957) [Sov. Phys. JETP 5, 1174 (1957)].

[18] W. T. M. Irvine, Moreau's hydrodynamic helicity and the life of vortex knots and links, C. R. Méch. 346, 170 (2018).

[19] M. R. Dennis, R. P. King, B. Jack, K. O’Holleran, and M. J. Padgett, Isolated optical vortex knots, Nat. Phys. 6, 118 (2010).

[20] J. F. Nye, Lines of circular polarization in electromagnetic wave fields, Proc. R. Soc. A 389, 279 (1983).

[21] P. G. de Gennes and J. Prost, The Physics of Liquid Crystals, 2nd ed. (Oxford University Press, Oxford, 1993).

[22] T. Machon and G. P. Alexander, Global defect topology in nematic liquid crystals, Proc. R. Soc. A 472, 20160265 (2016).

[23] T. Machon and G. P. Alexander, Umbilic Lines in Orientational Order, Phys. Rev. X 6, 011033 (2016).

[24] J.-S. B. Tai and I. I. Smalyukh, Three-dimensional crystals of adaptive knots, Science 365, 1449 (2019).

[25] See Supplemental Material at http://link.aps.org/ supplemental/10.1103/PhysRevLett.125.047801 for additional illustrations and examples, as well as further technical details.

[26] G. P. Alexander, Topology in liquid crystal phases, edited by S. Gupta and A. Saxena, in The Role of Topology in Materials (Springer, Cham, 2018).

[27] T. Machon, The topology of knots and links in nematics, Liq. Cryst. Today 28, 58 (2019).

[28] T. J. Machon, Aspects of geometry and topology in liquid crystalline phases, Ph.D. thesis, University of Warwick, 2016, http://webcat.warwick.ac.uk/record=b2870349 S1.

[29] J. V. Selinger, Interpretation of saddle-splay and the OseenFrank free energy in liquid crystals, Liq. Cryst. Rev. 6, 129 (2018).

[30] E. I. Kats and V. V. Lebedev, Landau theory for helical nematic phases, JETP Lett. 100, 110 (2014).

[31] G. Pająk, L. Longa, and A. Chrzanowska, Nematic twistbend phase in an external field, Proc. Natl. Acad. Sci. U.S.A. 115, E10303 (2018).

[32] R. D. Kamien, Liquids with chiral bond order, J. Phys. II (France) 6, 461 (1996).

[33] Z. Parsouzi et al., Fluctuation Modes of a Twist-Bend Nematic Liquid Crystal, Phys. Rev. X 6, 021041 (2016).

[34] C. Meyer and I. Dozov, Local distortion energy and coarsegrained elasticity of the twist-bend nematic phase, Soft Matter 12, 574 (2016).

[35] L. Radzihovsky and T. C. Lubensky, Nonlinear smectic elasticity of helical state in cholesteric liquid crystals and helimagnets, Phys. Rev. E 83, 051701 (2011).

[36] R. D. Kamien and R. A. Mosna, The topology of dislocations in smectic liquid crystals, New J. Phys. 18, 053012 (2016).

[37] H. K. Moffatt and R. L. Ricca, Helicity and the Călugăreanu invariant, Proc. R. Soc. A 439, 411 (1992).

[38] I. Dozov and C. Meyer, Analogy between the twist-bend nematic and the smectic A phases and coarse-grained description of the macroscopic $N_{T B}$ properties, Liq. Cryst. 44, 4 (2017).

[39] M. T. Murachver et al., Indication of a twist-grainboundary-twist-bend phase of flexible core bent-shape chiral dimers, Soft Matter 15, 3283 (2019).

[40] E. A. Matsumoto, R. D. Kamien, and G. P. Alexander, Straight round the twist: Frustration and chirality in smectics-A, Interface Focus 7, 20160118 (2017).

[41] M. Kleman and K. S. Krishnamurthy, Defects in the twistbend nematic phase: Stabilities and instabilities of focal conic domains and related topics, Phys. Rev. E 98, 032705 (2018).

[42] G. P. Alexander, B. G. Chen, E. A. Matsumoto, and R. D. Kamien, Power of the Poincaré Group: Elucidating the Hidden Symmetries in Focal Conic Domains, Phys. Rev. Lett. 104, 257802 (2010).

[43] D. Foster, C. Kind, P. J. Ackerman, J. S. B. Tai, M. R. Dennis, and I. I. Smalyukh, Two-dimensional skyrmion bags in liquid crystals and ferromagnets, Nat. Phys. 15, 655 (2019).

[44] P. J. Ackerman, R. P. Trivedi, B. Senyuk, J. van de Lagemaat, and I. I. Smalyukh, Two-dimensional skyrmions and other solitonic structures in confinement-frustrated chiral nematics, Phys. Rev. E 90, 012505 (2014).

[45] P. J. Ackerman and I. I. Smalyukh, Static three-dimensional topological solitons in fluid chiral ferromagnets and colloids, Nat. Mater. 16, 426 (2017).

[46] A. Duzgun, J. V. Selinger, and A. Saxena, Comparing skyrmions and merons in chiral liquid crystals and magnets, Phys. Rev. E 97, 062706 (2018).

[47] P. Sutcliffe, Skyrmion Knots in Frustrated Magnets, Phys. Rev. Lett. 118, 247203 (2017).

[48] S. Afghah and J. V. Selinger, Theory of helicoids and skyrmions in confined cholesteric liquid crystals, Phys. Rev. E 96, 012708 (2017).

[49] H. Geiges, An Introduction to Contact Topology (Cambridge University Press, Cambridge, England, 2008).

[50] M. V. Berry and J. H. Hannay, Umbilic points on Gaussian random surfaces, J. Phys. A 10, 1809 (1977).

[51] J. Etnyre and R. Ghrist, Gradient flows within plane fields, Commentarii mathematici Helvetici 74, 507 (1999).

[52] G. P. Alexander, B. G. Chen, E. A. Matsumoto, and R. D. Kamien, Colloquium: Disclination loops, point defects, and all that in nematic liquid crystals, Rev. Mod. Phys. 84, 497 (2012).

[53] P. J. Ackerman and I. I. Smalyukh, Diversity of Knot Solitons in Liquid Crystals Manifested by Linking of Preimages in Torons and Hopfions, Phys. Rev. X 7, 011006 (2017).

[54] B. G. Chen, P. J. Ackerman, G. P. Alexander, R. D. Kamien, and I. I. Smalyukh, Generating the Hopf Fibration Experimentally in Nematic Liquid Crystals, Phys. Rev. Lett. 110, 237801 (2013).

[55] P. Sutcliffe, Hopfions in chiral magnets, J. Phys. A 51, 375401 (2018).

[56] G. Călugăreanu, Sur les classes d'isotopie des nœuds tridimensionnels et leurs invariants, Czech. Math. J. 11, 588 (1961).

[57] F. B. Fuller, The writhing number of a space curve, Proc. Natl. Acad. Sci. U.S.A. 68, 815 (1971). 
[58] J. Binysh and G. P. Alexander, Maxwell's theory of solid angle and the construction of knotted fields, J. Phys. A 51, 385202.

[59] X.Z. Yu, W. Koshibae, Y. Tokunaga, K. Shibata, Y. Taguchi, N. Nagaosa, and Y. Tokura, Transformation between meron and skyrmion topological spin textures in a chiral magnet, Nature (London) 564, 95 (2018).

[60] V. I. Arnold and B. A. Khesin, Topological Methods in Hydrodynamics (Springer-Verlag, New York, 1998).

[61] R. E. Gompf and A. I. Stipsicz, 4-Manifolds and Kirby Calculus (American Mathematical Society, Providence, 1999).
[62] C. A. Whitfield, T. C. Adhyapak, A. Tiribocchi, G. P. Alexander, D. Marenduzzo, and S. Ramaswamy, Hydrodynamic instabilities in active cholesteric liquid crystals, Eur. Phys. J. E 40, 50 (2017).

[63] J. Binysh, Ž. Kos, S. Čopar, M. Ravnik, and G. P. Alexander, Three-Dimensional Active Defect Loops, Phys. Rev. Lett. 124, 088001 (2020).

[64] G. Duclos et al., Topological structure and dynamics of three-dimensional active nematics, Science 367, 1120 (2020). 